Nephrologe 2007 2 2:6-13

DOI 10.1007/s11560-006-0050-2

Online publiziert: 15 . Dezember 2006

(c) Springer Medizin Verlag 2006

\section{Redaktion}

R.P. Wüthrich, Zürich
D. Varga ${ }^{1} \cdot$ M. Körner ${ }^{2} \cdot$ H.-P. Marti ${ }^{1}$

${ }^{1}$ Klinik und Poliklinik für Nephrologie und Hypertonie, Inselspital Bern

${ }^{2}$ Institut für Pathologie, Universität Bern
Die fortgeschrittene Niereninsuffizienz stellt für die Betroffenen eine schwere Belastung dar. Die Nierenersatztherapie ist zwar lebensrettend, bedeutet für den Patienten jedoch eine einschneidende Veränderung seiner Lebensgewohnheiten und eine deutliche Beeinträchtigung seiner Lebensqualität.

Glomeruläre entzündliche Erkrankungen - die Glomerulonephritiden im engeren Sinn - liegen in westlichen Ländern mit etwa $15 \%$ an 3 . Stelle der führenden Ursachen eines fortgeschrittenen Nierenversa-

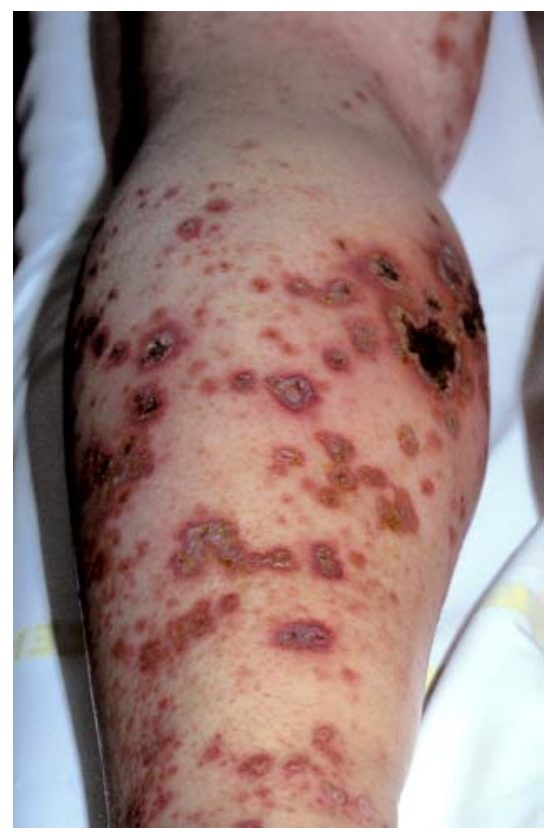

Abb. 1 A Allopurinol-induzierte, nekrotisierende Vaskulitis der Haut

\title{
Diagnostik bei Glomerulonephritis
}

\section{Rationaler Abklärungsgang}

gens, nur übertroffen von diabetischer $\mathrm{Ne}$ phropathie und Nephroangiosklerose als Folge von arterieller Hypertonie $[11,19]$. Eine korrekte und frühzeitige Behandlung kann in manchen Fällen die Entwicklung einer dialysepflichtigen Niereninsuffizienz verzögern oder gar verhindern, weshalb die rasche und genaue Diagnosestellung entscheidend zur Verbesserung der Prognose beiträgt $[14,15]$.

\section{Klinische Präsentation}

Glomerulonephritiden gehen mit entzündlichen, d. h. strukturellen und funktionellen Alterationen der Glomeruli einher. Pathogenetisch spielt das Immunsystem eine zentrale Rolle [20]. Von den eigentlichen Glomerulonephritiden abgrenzbar sind die primär nicht entzündlichen Glomerulopathien, wie beispielsweise die diabetische Nephropathie oder die Amyloidose mit glomerulärem Befall. Diese Unterscheidung ist aber nicht immer klar, und es gibt Überschneidungen.

Die Glomerulonephritiden können sich akut, chronisch, weitgehend asymptomatisch oder mit ausgeprägten klinischen Zeichen und Beschwerden äußern [8]. Die - Tab. 1 fasst die wichtigsten klinischen Erscheinungsformen der Glomerulonephritiden zusammen. Die rezidivierende Makrohämaturie kann meist der IgA-Nephropathie (evtl. im Rahmen einer HenochSchönlein-Purpura) zugeordnet werden.

Im Zentrum der Befunde stehen Hypertonie, Ödeme, Proteinurie und Hämaturie sowie Niereninsuffizienz (Ab- nahme der glomerulären Filtrationsrate mit erhöhtem Kreatinin im Serum/Plasma). Hinzu kommen Symptome allfälliger Systemerkrankungen, wie z. B. leukozytoklastische Vaskulitis mit Purpura der Haut, oder Arthritiden.

Zur Definition des nephrotischen Syndroms gehören Proteinurie, Ödeme, Hypoalbuminämie, Hyperlipidämie und Lipidurie $[8,11]$. In der klinischen Praxis wird für ein nephrotisches Syndrom meistens eine 24-h-Proteinurie $>3 \mathrm{~g}$ verlangt; andere Definitionen sprechen von $>3 \mathrm{~g} / 1,73 \mathrm{~m}^{2}$ oder $>3,5 \mathrm{~g} / 1,73 \mathrm{~m}^{2}[11,17]$.

Eine Proteinurie im nephrotischen Bereich spiegelt definitiv eine glomeruläre Schädigung wider und ist ein ungünstiges prognostisches Zeichen $[6,8]$. Wichtige Risikofaktoren für eine raschere Progression einer Nephropathie nebst Proteinurie sind arterielle Hypertonie, Niereninsuffizienz, Nikotinkonsum und männliches Geschlecht $[3,5,6,11,15,17]$.

\section{Abklärungsgang}

Glomerulonephritiden können mannigfaltige Ätiologien aufweisen. Die meisten Formen dieser Erkrankungen manifestieren sich als erworbene, in der Regel immunologisch bedingte Störungen, die idiopathisch oder aufgrund erkennbarer Ursachen im Verlauf des Lebens auftreten.

Die Glomerulonephritiden können familiär gehäuft (z. B. fokal-segmentale Glomerulosklerose (FSGS) oder IgA-Nephropathie) oder kongenital (z. B. memb- 
Hier steht eine Anzeige.

黛 Springer 

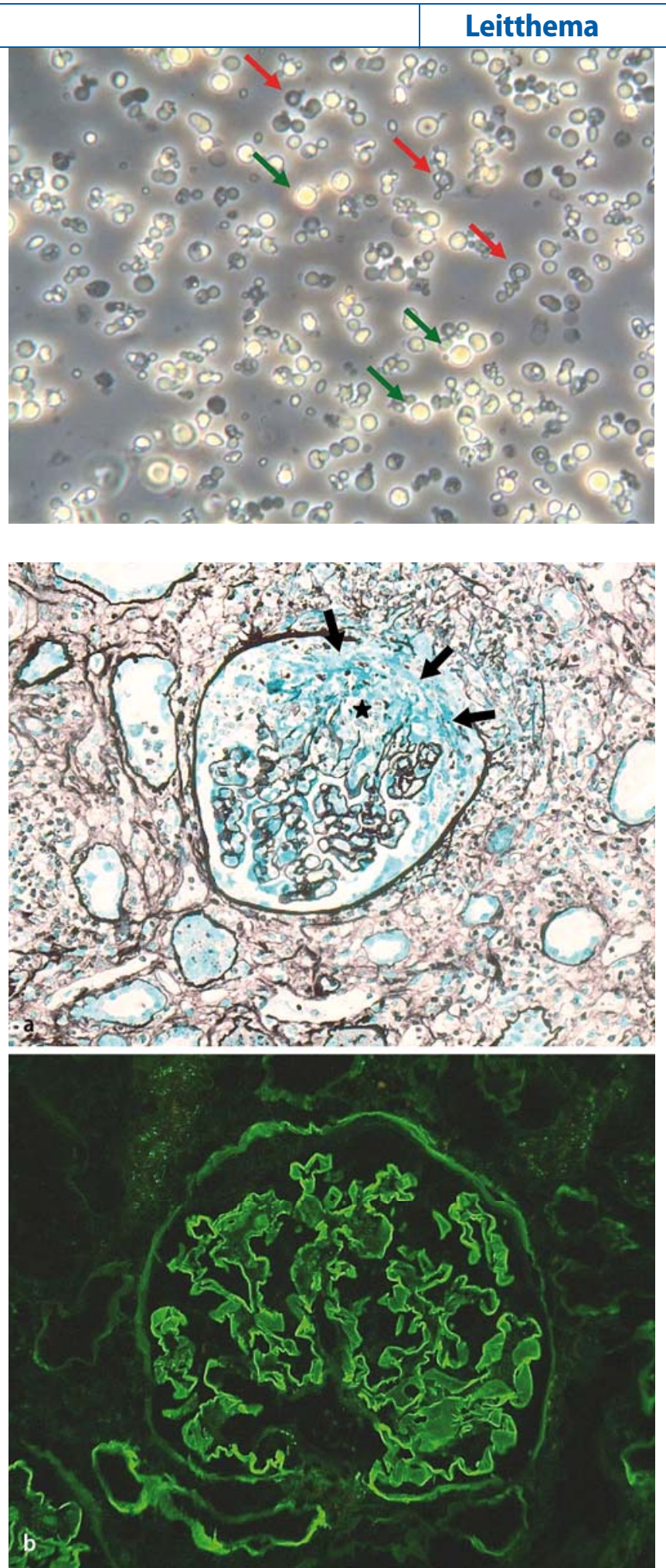

Abb. $2<$ Glomeruläre Hämaturie in der Urinuntersuchung mittels Phasenkontrastmikroskopie. Grüne Pfeile: intakte, glatt begrenzte Erythrozyten. Rote Pfeile: dysmorphe, glomeruläre Erythrozyten (Ruth Russi, Foto PH 40×10)

Abb. $3<$ Anti-Glomeruläre Basalmembranantikörper-vermittelte Glomerulonephritis. a Glomerulus mit segmentaler Nekrose (Stern) und angrenzendem zellulärem Halbmond (Pfeile) (Silber-Methenamin Vergr. 200:1). b Lineare Positivität der glomerulären Basalmembran für lgG (Anti-lgG-Immunfluoreszenz, Vergr. 200:1)

ranöse Nephropathie als Folge von antiEndopeptidase-Antikörpern) auftreten $[4,8,12,18]$. Ferner können glomeruläre entzündliche Erkrankungen als primäre Glomerulonephritiden auf die Niere beschränkt ablaufen oder als sekundäre Glomerulonephritiden im Rahmen von Systemerkrankungen auftreten.
Zusammen mit den klinischen Zeichen bedingt die vielfältige Pathogenese den rationalen Abklärungsgang dieser Erkrankungen. In diesem Zusammenhang ist ein nicht nur auf die Niere begrenzter systemischer Ansatz, beginnend mit sorgfältiger Anamnese und klinischer Untersuchung, unerlässlich. Die wichtigsten
Laboruntersuchungen und bildgebenden Abklärungen sind in der $\bullet$ Tab. 2 zusammengefasst.

Besonders die rasch progrediente Glomerulonephritis (nekrotisierende Glomerulonephritis mit Halbmondbildung) muss in diesem Zusammenhang als Notfall betrachtet und entsprechend dringlich abgeklärt und behandelt werden (• Tab. 2, 3). Ansonsten besteht die Gefahr der irreversiblen Zerstörung der Glomeruli mit Sklerosierung und Funktionsverlust innerhalb weniger Wochen bis Monate.

\section{Anamnese}

Bei der Erhebung der Anamnese ist besonders auf folgende Punkte zu achten: Sehr wichtig ist die Exposition von Medikamenten. Die Einnahme von nichtsteroidalen Antirheumatika (NSAR) kann zu Glomerulonephritiden wie „minimalchange“ Nephropathie oder membranöser Glomerulonephritis führen [5]. Allopurinol kann in seltenen Fällen zu einer schweren nekrotisierenden Vaskulitis der Haut mit Nierenbefall im Sinn einer begleitenden Glomerulonephritis führen, wie anhand einer eigenen Patientenbeobachtung in $\bullet$ Abb. 1 dargestellt. Grundsätzlich muss jedes Medikament hinsichtlich solcher renaler Nebenwirkungen in Erwägung gezogen werden.

Akute und chronische Infekte bakterieller (z. B. Streptokokken) oder besonders viraler (z. B. Hepatitis B/C, HIV) Ursache können mit Glomerulonephritiden assoziiert sein.

Insbesondere beim Vorliegen von Allgemeinsymptomen wie Fieber, Inappetenz, Müdigkeit und Gewichtsverlust müssen systemische Vaskulitiden, Kollagenosen und maligne Tumoren gesucht werden. Speziell sollte in diesem Zusammenhang auch nach blutig tingiertem Nasensekret oder Auswurf gefragt werden - dies als Hinweise für die besonders gefährlichen pulmorenalen Syndrome etwa im Rahmen einer ANCA-positiven Vaskulitis oder des Goodpasture-Syndroms.

Die Familienanamnese muss beachtet werden, da familiäre oder vererbte Formen der Glomerulonephritiden ebenfalls gesucht werden müssen. Beispiele sind das Alport-Syndrom oder familiär auftretende Glomerulonephritiden wie IgA-Ne- 
phropathie, hämolytisch-urämisches Syndrom oder fokal-segmentale Glomerulosklerose (FSGS; $[5,18]$ ).

\section{Klinische Untersuchung}

Eine arterielle Hypertonie ist ein wichtiges, aber nicht obligates Zeichen, das besonders bei akuten Glomerulonephritiden mit nephritischem Verlauf oder bei chronischen Formen mit ausgeprägter Niereninsuffizienz und Hypervolämie auftritt. Bezüglich der Evaluation der Hypertonie gehört - neben der Abklärung der Nephropathie - die Suche nach hypertensiver Retinopathie und linksventikulärer Hypertrophie als weitere Endorganschäden dazu.

\section{- Die Ödembildung steht beim nephrotischen Syndrom klinisch im Vordergrund.}

Typischerweise treten initial morgendliche Lidödeme, gefolgt von Ödemen in den Extremitäten sowie später Aszites und Pleuraergüsse auf. Allerdings kommt es grundsätzlich bei allen Glomerulonephritiden mit deutlicher Niereninsuffizienz im Verlauf zur Bildung von Ödemen. Eine asymmetrische Beinschwellung kann aber ein Zeichen einer tiefen Venenthrombose darstellen, besonders bei Patienten mit nephrotischem Syndrom und Hyperkoagulabilität.

Schließlich müssen auch Zeichen von malignen, infektiösen oder entzündlichen Systemerkrankungen gesucht werden, wie z. B. Hauteffloreszenzen, Arthritiden, Mononeuritis multiplex, vergrößerte Lymphknoten oder Hepatosplenomegalie.

\section{Laboruntersuchungen}

Die Standarddiagnostik im Blut und Urin gemäß $\bullet$ Tab. 2 folgt der Anamnese und der körperlichen Untersuchung.

Die Hämaturie als Folge einer Glomerulonephritis ist charakterisiert durch viele dysmorphe Erythrozyten, wie in $\bullet$ Abb. 2 dargestellt. Für diese zudem sehr untersucherabhängige Analyse wird ein Phasenkontrastmikroskop benötigt. Im klinischen Alltag kann davon ausgegangen werden, dass es sich um eine glomeruläre Hämaturie handelt, wenn die Hämaturie

Nephrologe 2007 ·2:6-13 DOI 10.1007/s11560-006-0050-2

(c) Springer Medizin Verlag 2006

D. Varga $\cdot$ M. Körner $\cdot$ H.-P. Marti Diagnostik bei Glomerulonephritis. Rationaler Abklärungsgang

Zusammenfassung

Glomerulonephritiden sind eine wichtige und häufige Ursache der fortgeschrittenen Niereninsuffizienz. Hinsichtlich Therapierbarkeit und Prognose sind eine frühzeitige $\mathrm{Ab}$ klärung und Diagnosestellung von entscheidender Bedeutsamkeit. Die wichtigsten klinischen Zeichen der Glomerulonephritis per se sind arterielle Hypertonie, Ödeme, Proteinurie, Hämaturie und Niereninsuffizienz. Dazu kommen Symptome allfälliger Systemerkrankungen. Der Abklärungsgang umfasst Erhebung der Anamnese, klinische Untersuchung, Laboruntersuchungen im Blut und Urin, bildgebende Verfahren wie Sonographie und Magnetresonanzuntersuchung und in den meisten Fällen die perkutane Nierenbiopsie. Bei der Diagnostik ist es besonders wichtig eine behandelbare Ursache - wie Medikamentennebenwirkung, Infekt, Tumor oder entzündliche Systemerkrankung - zu erkennen. Glomerulonephritiden können einerseits aufgrund ihrer klinischen Präsentation und andererseits basierend auf den histologischen Veränderungen genauer klassifiziert werden.

\section{Schlüsselwörter}

Glomerulonephritis · Glomerulopathie · Proteinurie $\cdot$ Hämaturie $\cdot$ Niereninsuffizienz

\section{Diagnostic evaluation of glomerulonephritis. Effective diagnostic strategy}

\section{Abstract}

Glomerulonephritides represent an important and frequent cause of end-stage renal disease. Early detection and diagnostic evaluation are crucial for the determination of therapy and prognosis. The most important clinical features of glomerulonephritis are arterial hypertension, edema formation, proteinuria, hematuria and renal failure. In addition, there may be signs and symptoms of underlying systemic disease. The diagnostic evaluation of glomerulonephritis contains careful history taking, physical examination, laboratory analyses of blood and urine, and radiological investigations such as ultrasound and magnetic resonance imaging. In addition, the final diagnosis often requires a kidney biopsy. In terms of the diagnostic workup of glomerulonephritis, it is of great importance to detect a possible reversible cause of the disease, such as the side-effects of medication, infection, malignancy or systemic inflammatory disease. Finally, the classification of glomerulonephritis can be based on clinical presentation and histological features.

\section{Keywords}

Glomerulonephritis · Glomerulopathy · Proteinuria $\cdot$ Hematuria $\cdot$ Kidney failure 
Tab. 1 Sechs klinische Erscheinungsbilder der Glomerulonephritiden. (Mod. nach [5])

\begin{tabular}{|c|c|c|}
\hline Kategorie & Klinisches Bild & Laborbefunde (Urin/Blut) \\
\hline $\begin{array}{l}\text { Asymptomatische Urin- } \\
\text { abnormalitäten }\end{array}$ & $\begin{array}{l}\text { Normale Nierenfunktion, keine } \\
\text { Ödeme oder arterielle Hypertonie }\end{array}$ & $\begin{array}{l}\text { Proteinurie im nichtnephro- } \\
\text { tischen Bereich }(<3 \mathrm{~g} / \mathrm{Tag}) \\
\text { und/oder mikroskopische } \\
\text { Hämaturie }\end{array}$ \\
\hline $\begin{array}{l}\text { Rezidivierende makros- } \\
\text { kopische Hämaturie }\end{array}$ & $\begin{array}{l}\text { Schmerzlose Episoden von Makrohä- } \\
\text { maturie, oft mit Infektionen assoziiert, } \\
\text { dazwischen asymptomatische Urin- } \\
\text { befunde }\end{array}$ & $\begin{array}{l}\text { Hämaturie mit/ohne Protein- } \\
\text { urie wechselnden Ausmaßes }\end{array}$ \\
\hline Nephrotisches Syndrom & $\begin{array}{l}\text { Ödeme, arterielle Hypertonie nicht } \\
\text { obligat, Prädisposition zu venösen } \\
\text { Thromboembolien und Infektionen }\end{array}$ & $\begin{array}{l}\text { Proteinurie im nephrotischen } \\
\text { Bereich ( } \geq 3 \mathrm{~g} / \mathrm{Tag}) \text {, Hypal- } \\
\text { buminämie, Hyperlipidämie, } \\
\text { Kreatinin normal oder erhöht }\end{array}$ \\
\hline Nephritisches Syndrom & $\begin{array}{l}\text { Akute Nierenfunktionseinschränkung } \\
\text { mit Salz- und Wasserretention, Öde- } \\
\text { men und arterieller Hypertonie }\end{array}$ & $\begin{array}{l}\text { Hämaturie mit Erythrozyten- } \\
\text { zylindern und Proteinurie im } \\
\text { nichtnephrotischen Bereich }\end{array}$ \\
\hline $\begin{array}{l}\text { Rasch progrediente } \\
\text { Glomerulonephritis }\end{array}$ & $\begin{array}{l}\text { Rasch zunehmende Niereninsuffi- } \\
\text { zienz, evtl. mit zusätzlichen Symp- } \\
\text { tomen einer Vaskulitis oder Kollage- } \\
\text { nose (z. B. Haut, Gelenke, Lunge) }\end{array}$ & $\begin{array}{l}\text { Kreatininanstieg innerhalb } \\
\text { Tagen bis wenigen Wochen, } \\
\text { Mikrohämaturie und oft nicht- } \\
\text { nephrotische Proteinurie }\end{array}$ \\
\hline $\begin{array}{l}\text { Chronische Glomerulo- } \\
\text { nephritis }\end{array}$ & $\begin{array}{l}\text { Langsam progrediente Nieren- } \\
\text { funktionseinschränkung, arterielle } \\
\text { Hypertonie }\end{array}$ & $\begin{array}{l}\text { Persistierende Proteinurie mit } \\
\text { oder ohne Mikrohämaturie, } \\
\text { langsam zunehmende Erhö- } \\
\text { hung des Kreatinins über viele } \\
\text { Jahre }\end{array}$ \\
\hline
\end{tabular}

Tab. 2 Abklärungen bei Glomerulonephritis

\section{Standardlabor}

Blut

BSR, CRP, weißes und rotes Blutbild, Haptoglobin, freies Hämoglobin, Gerinnung (Quick-INR, PTT, TZ)

Natrium, Kalium, Kalzium, Phosphat, Glukose (HbA1c), Harnstoff, Kreatinin, Gesamtprotein, Albumin, Bilirubin, ALAT, ASAT, $\gamma \mathrm{GT}$, $\mathrm{AP}, \mathrm{CK}, \mathrm{LDH}$, Cholesterin, Triglyzeride

Hepatitis-B/C- und HIV-Serologie, Blutkulturen

\section{Urin}

Urinstatus (inklusive Mikroskopie), Urinkultur, Spoturin (Protein/Kreatinin), 24-hUrin (Kreatininclearance, Protein, Albumin)

Urinstatus (Suche nach dysmorphen Erythrozyten), 24-h-Urin (Bence-Jones-Proteinurie)

\section{Histologie}

Nierenbiopsie

\section{Radiologische Abklärungen}

\section{Konventionelle Aufnahmen}

Thorax p/a und lateral

\section{Sonographie}

Abdomensonographie, Duplexsonographie der Nieren

\section{Magnetresonanztomo-} graphie (MRI) Angio-MRI der Nieren und Nierenarterien

Beim Fehlen von Risikofaktoren wie Proteinurie, Hypertonie oder Niereninsuffizienz sowie in Abwesenheit einer Systemerkrankung oder positiven Familienanamnese kann mit einer Nierenbiopsie noch abgewartet werden $[11,17]$.

Bezüglich der Bestimmung der Proteinurie genügt in der Praxis die Messung des Protein-Kreatinin-Quotienten in einer Einzelprobe von Spontanurin $[7,11]$. Dabei wird die Proteinausscheidung über $24 \mathrm{~h}$ in Gramm berechnet; aus dem gemessenen Protein- (g)/Kreatinin- (mmol) Quotienten, multipliziert mit der errechneten täglichen Kreatininausscheidung (Berechnung mit der Formel nach Cockcroft und Gault; [7, 11]).

Erhärtet sich anschließend der Verdacht auf eine Glomerulonephritis und bleibt insbesondere deren Genese unklar, erfolgen die Zusatzuntersuchungen, wie ebenfalls in $\bullet$ Tab. 2 dargestellt.

In Spezialfällen können diese Abklärungen beispielsweise bei jüngeren $\mathrm{Pa}$ tienten (Kindern) mit (therapierefraktärer) fokal-segmentaler Glomerulosklerose (FSGS) durch genetische Analysen wie der Suche nach Mutationen in Proteinen der Podozyten (z. B. Podocin) ergänzt werden $[9,18]$.

\section{Radiologische Untersuchungen}

Mittels einer konventionellen Thoraxaufnahme sollte insbesondere nach Lungeninfiltraten (Differenzialdiagnose: Infekt, Malignom oder insbesondere bei bilateralem Befall auch Blutung), Lungenödem, vergrößerten Lymphnoten und Pleuraergüssen gesucht werden. In der Regel, insbesondere bei entsprechender Risikokonstellation (z. B. ältere Patienten, Patienten mit membranöser oder „minimal-change“ Nephropathie), wird mit einer Abdomensonographie durch die Darstellung von Leber, Milz und Lymphknoten nach einem Malignom gefahndet; evtl. können diese Abklärungen noch durch eine Kolonoskopie ergänzt werden $[1,11,16]$.

Die Duplexsonographie der Nieren analysiert und bestimmt in 1. Linie folgende Parameter: Nierengröße, Echodichte der Nierenrinde (erhöht bei Glomerulonephritis), Missbildungen (z. B. Zysten bei autosomal dominanter polyzystischer Nierenerkrankung, ADPKD),

mit Proteinurie und evtl. Erythrozytenzylindern koexistiert. Eine gewisse Unsicherheit besteht in der Weiterabklärung von Patienten mit isolierter, asymptomatischer Hämaturie nach Ausschluss von Urinin- fektion, Trauma, Urogenitaltumor oder Kristallurie (Kalzium, Harnsäure oder Oxalat) mit und ohne Steinleiden [11]. Besonders bei jüngeren Patienten hat eine beträchtliche Anzahl eine IgA-Nephropathie. 


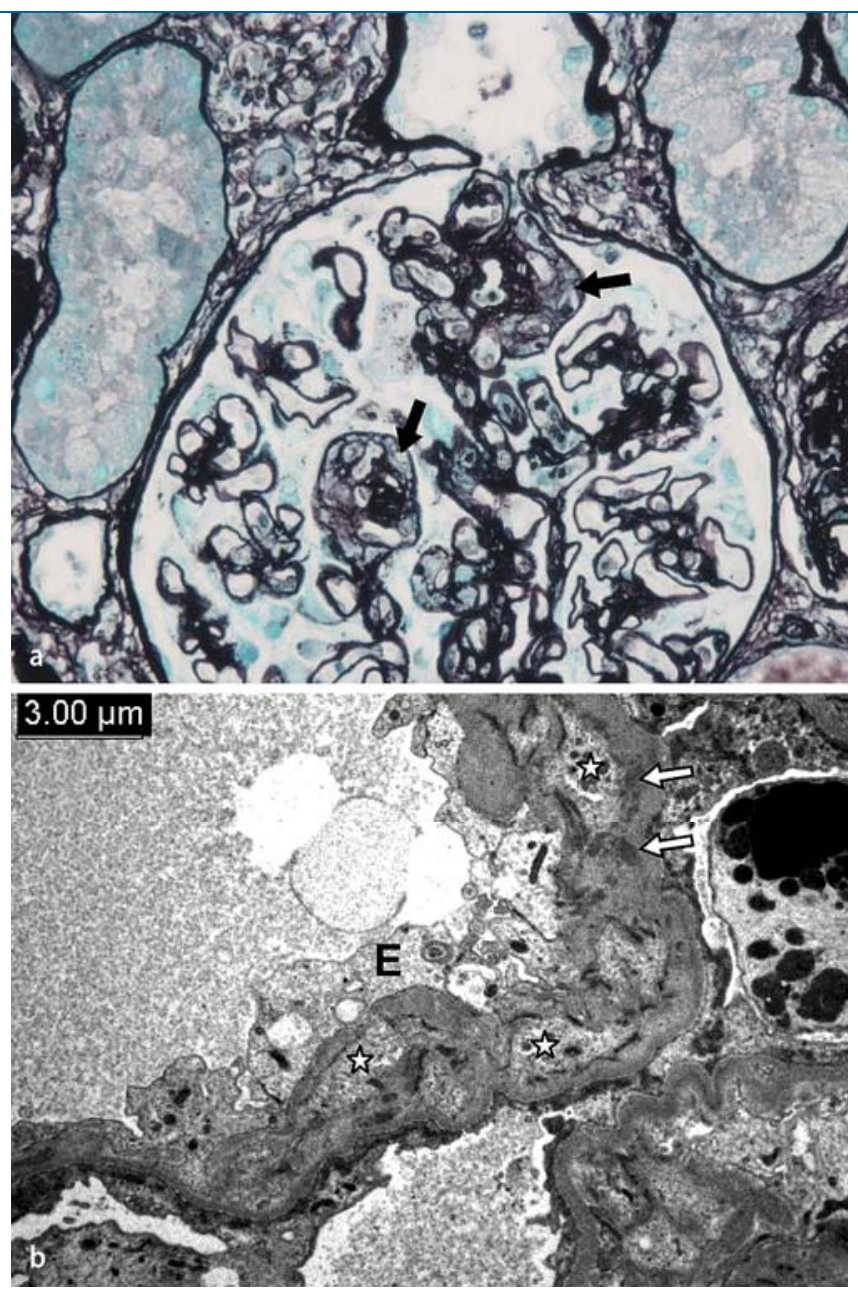

Abb. 4 \ Segmentale Lupusglomerulonephritis. a Verbreiterte Kapillarschlingen (Pfeile) mit Basalmembranneubildung im Sinn eines membranoproliferativen Reaktionsmusters (Silbermethenamin, Vergr. 400:1). b Die entsprechende Elektronenmikroskopie zeigt subendotheliale Depots (Pfeile), einen verbreiterten subendothelialen Raum (Sterne) und eine Basalmembranneubildung unter dem Endothel $(E)$
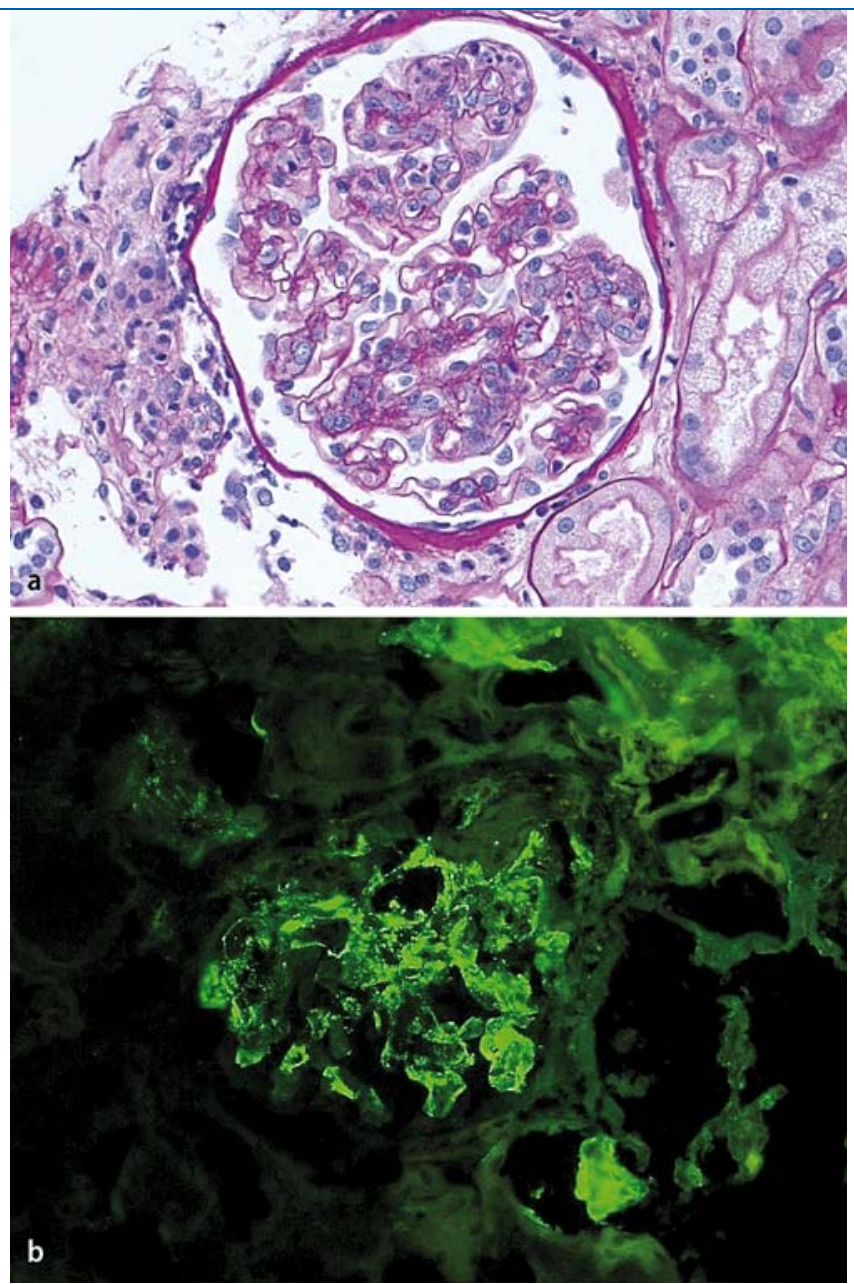

Abb. $5 \Delta$ Purpura Schönlein-Henoch. a Diffuse mesangial und endokapillär proliferative Glomerulonephritis (PAS, Vergr. 400:1). b Mesangiale lgAAblagerungen (Anti-IgA-Immunfluoreszenz, Vergr. 200:1)
Abfluss des Urins (z. B. Ausschluss einer Harnwegsobstruktion mit gestautem Nierenbeckenkelchsystem), Durchblutung des Parenchyms und Blutfluss in den Nierenarterien (Ausschluss von Nierenarterienstenosen, die zu einer arteriellen $\mathrm{Hy}$ pertonie, Niereninsuffizienz und Proteinurie sogar selten im nephrotischen Bereich führen können).

In speziellen Fällen, insbesondere auch bei schwer untersuchbaren, oft adipösen Patienten oder bei nichtkonklusiver Sonographie kann auch eine Angiographie mittels Computertomographie oder Magnetresonanztomographie veranlasst werden [Ausschluss von Nierenarterienstenosen, wie oben erwähnt, sowie Suche nach arteriellen Aneurysmen bei Verdacht auf Polyarteritis nodosa (PAN)].

\section{Nierenbiopsie}

Die klinischen Befunde und die Laboruntersuchungen lassen in der Regel mit wenigen Ausnahmen wie der diabetischen Nephropathie - keine eindeutigen Rückschlüsse auf den histologischen Befund einer Glomerulopathie zu. Dies betrifft auch das nephrotische Syndrom, obwohl dieses die übliche Präsentation bei "minimal-change“ Nephropathie, membranöser Nephropathie und fokal-segmentaler Glomerulosklerose (FSGS) darstellt.

Bei den meisten Patienten ist deshalb die perkutane, ultraschallgesteuerte Nierenbiopsie notwendig, um Art und Schwergrad der Glomerulonephritis zu bestimmen $[5,11,17]$. Erst daraus lassen sich genaue Rückschlüsse bezüglich me- dikamentöser Therapieindikation und Prognose ziehen. Eine exakte Diagnose ist auch wichtig hinsichtlich einer möglichen Nierentransplantation, da Glomerulonephritiden jeweils in variabler Häufigkeit und mit unterschiedlichen Folgen im Transplantat wieder auftreten können [2].

Die histologische Diagnosestellung sollte grundsätzlich frühzeitig erfolgen, idealerweise vor Entwicklung einer Niereninsuffizienz oder eines voll ausgebildeten nephrotischen Syndroms.

Die histologische Aufarbeitung der Nierenbiopsie ist komplex und beinhaltet Lichtmikroskopie (inklusive Immunhistologie), Immunfluoreszenzmikroskopie und Elektronenmikroskopie. Basierend auf diesen Analysen erfolgt die his- 
Tab. 3 Histologische Klassifizierung der Glomerulonephritiden. (Mod. nach [5])

\begin{tabular}{|c|c|c|c|c|}
\hline Histologischer Typ & Lichtmikroskopie & Immunfluoreszenz & Elektronenmikroskopie & Spezielle Beispiele \\
\hline $\begin{array}{l}\text { "Minimal-change" } \\
\text { Nephropathie }\end{array}$ & $\begin{array}{l}\text { Keine sichtbaren Verände- } \\
\text { rungen }\end{array}$ & $\begin{array}{l}\text { Keine Ablagerungen von Im- } \\
\text { munglobulinen (Ig) }\end{array}$ & $\begin{array}{l}\text { Verschmelzung der Fußfort- } \\
\text { sätze der Podozyten }\end{array}$ & NSAR-induzierte Nephropathie \\
\hline $\begin{array}{l}\text { Fokal-segmentale } \\
\text { Glomerulosklerose } \\
\text { (FSGS) }\end{array}$ & $\begin{array}{l}\text { Fokal-segmentale Kapillar- } \\
\text { sklerose bzw. Hyalinose }\end{array}$ & $\begin{array}{l}\text { IgM- und Komplement-C3-Ab- } \\
\text { lagerungen in den betroffenen } \\
\text { Bezirken }\end{array}$ & Mesangiale Sklerose & $\begin{array}{l}\text { NSAR-induzierte Nephropathie, } \\
\text { HIV-assoziierte Nephropathie }\end{array}$ \\
\hline $\begin{array}{l}\text { Membranöse Ne- } \\
\text { phropathie }\end{array}$ & $\begin{array}{l}\text { Verdickung der glomerulären } \\
\text { Basalmembran ohne klare } \\
\text { Zellproliferation }\end{array}$ & $\begin{array}{l}\text { Granuläre Anfärbung der } \\
\text { glomerulären Basalmembran } \\
\text { durch Ig und Komplement- } \\
\text { faktoren }\end{array}$ & $\begin{array}{l}\text { Subepitheliale Immundepots, } \\
\text { dazwischen Ausläufer der } \\
\text { glomerulären Basalmembran } \\
\text { („spikes“) }\end{array}$ & $\begin{array}{l}\text { SLE-Nephritis ( } \bullet \text { Abb. } 4) \\
\text { Hepatitis-B-assoziierte Glome- } \\
\text { rulonephritis }\end{array}$ \\
\hline $\begin{array}{l}\text { Mesangioprolife- } \\
\text { rative Glomerulo- } \\
\text { nephritis }\end{array}$ & $\begin{array}{l}\text { Vermehrung der mesangi- } \\
\text { alen und teilweise der endo- } \\
\text { thelialen Zellen }\end{array}$ & $\begin{array}{l}\text { Immunkomplex-Ablage- } \\
\text { rungen (IgG, IgA, IgM und C3- } \\
\text { Komplement) }\end{array}$ & $\begin{array}{l}\text { Große noduläre Immunde- } \\
\text { pots (Humps) subepithelial; } \\
\text { mesangiale Depots }\end{array}$ & $\begin{array}{l}\text { IgA-Nephropathie (Henoch- } \\
\text { Schönlein Purpura; • Abb. 5), } \\
\text { Postinfektiöse Glomeruloneph- } \\
\text { ritis, SLE-Nephritis }\end{array}$ \\
\hline $\begin{array}{l}\text { Membranoprolife- } \\
\text { rative Glomerulo- } \\
\text { nephritis }\end{array}$ & $\begin{array}{l}\text { Mesangiale Zell- und Matrix- } \\
\text { vermehrung, Verdickung der } \\
\text { glomerulären Basalmembran } \\
\text { (Doppelkonturen) }\end{array}$ & $\begin{array}{l}\text { Vorwiegend C3 mesangial so- } \\
\text { wie an Kapillarschlingen, z. T. } \\
\text { auch Ig und C4 }\end{array}$ & $\begin{array}{l}\text { Subendotheliale Immun- } \\
\text { depots (Typ I) oder „Dense } \\
\text { Deposit Disease“ (Typ II) }\end{array}$ & $\begin{array}{l}\text { Hepatitis-C-assoziierte Glome- } \\
\text { rulonephritis (Kryoglobulinä- } \\
\text { mie) }\end{array}$ \\
\hline $\begin{array}{l}\text { Nekrotisierende } \\
\text { Glomerulonephritis } \\
\text { und/oder Glome- } \\
\text { rulonephritis mit } \\
\text { Halbmondbildung }\end{array}$ & $\begin{array}{l}\text { Halbmondbildung durch } \\
\text { Zellproliferation (wie epithe- } \\
\text { liale Zellen und Makropha- } \\
\text { gen); Schlingennekrosen }\end{array}$ & $\begin{array}{l}\text { Ohne Immunablagerungen } \\
\text { („,pauci-immun“) oder Immun- } \\
\text { komplexe an der glomerulären } \\
\text { Basalmembran (linear oder } \\
\text { granulär) }\end{array}$ & $\begin{array}{l}\text { Meistens keine zusätzlichen } \\
\text { Aussagen }\end{array}$ & $\begin{array}{l}\text { M. Wegener, Mikroskopische } \\
\text { Polyangiitis Churg-Strauss-Syn- } \\
\text { drom, Goodpasture-Syndrom } \\
\text { (• Abb. 3) }\end{array}$ \\
\hline
\end{tabular}

tologische Klassifikation der Glomerulonephritiden, wie in der $\bullet$ Tab. 3 dargestellt. Diese Klassifikation unterscheidet zwischen primären (z. B. membranöse Nephropathie ohne Systemerkrankung) und sekundären (z. B. Glomerulonephritis mit Halbmondbildung beim Goodpasture-Syndrom oder SLE-Nephritis, wie in - Abb. 3 und 4 dargestellt) Formen der Glomerulonephritis und der Anwesenheit (z. B. diffuse mesangial und endokapillär proliferative Glomerulonephritis; - Abb.5) oder dem Fehlen (z. B. „minimal-change“ Nephropathie) von proliferativen Veränderungen im Glomerulus. In Zukunft kann diese histologische Klassifikation der Glomerulonephritiden mit der Transkriptomanalyse einer Vielzahl pathogenetisch relevanter Gene mittels cDNA-Microarrays wohl noch ergänzt und verfeinert werden $[10,13]$.

\section{Fazit für die Praxis}

- Der erstbehandelnde praktizierende Arzt spielt eine große Rolle bei der Früherkennung einer Glomerulonephritis.

- Die klinischen Symptome der Glomerulonephritiden bestehen sowohl aus wegweisenden Befunden (z. B. Proteinurie und Hämaturie) als auch aus unspezifischeren Zeichen (z. B. arterielle Hypertonie und Ödeme). Bei Patienten mit solchen Symptomen muss an eine Glomerulonephritis gedacht werden.

- Entsprechende Basisabklärungen können zu einem großen Teil in der Praxis erfolgen. Eine rechtzeitige Überweisung an den Facharzt ist anschließend notwendig, damit eine präzise Diagnose gestellt werden kann.

- Die Nierenbiopsie ist die beste Methode zur abschließenden Klassifikation der entsprechenden Glomerulonephritis. Die histologischen Befunde bilden die Basis für die folgende medizinische Behandlung und die Einschätzung der Prognose des Krankheitsverlaufs.

- Die rasch progrediente Glomerulonephritis ist in diesem Zusammenhang das wichtigste Krankheitsbild, das nicht verpasst werden darf.

\section{Korrespondierender Autor \\ Prof. Dr. H.-P. Marti

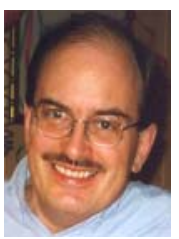 \\ Klinik und Poliklinik für Ne- phrologie und Hypertonie, Inselspital Bern 3010 Bern, Schweiz hans-peter.marti@insel.ch}

Interessenkonflikt. Es besteht kein Interessenkonflikt. Der korrespondierende Autor versichert, dass keine Verbindungen mit einer Firma, deren Produkt in dem Artikel genannt ist, oder einer Firma, die ein Konkurrenzprodukt vertreibt, bestehen. Die Präsentation des Themas ist unabhängig und die Darstellung der Inhalte produktneutral.

\section{Literatur}

1. Birkeland SA, Storm HH (2003) Glomerulonephritis and malignancy: a population-based analysis. Kidney Int 63: 716-721

2. Briganti EM, Russ GR, McNeil JJ et al. (2002) Risk of renal allograft loss from recurrent glomerulonephritis. N Engl J Med 347: 103-109

3. Couser WG (1999) Glomerulonephritis. Lancet 353: 1509-1515

4. Debiec H, Guigonis V, Mougenot B et al. (2002) Antenatal membranous glomerulonephritis due to anti-neutral endopeptidase antibodies. N Engl J Med 346: 2053-2060

5. Dendorfer U, Mann J (2006) Glomerulonephritis. Dtsch Med Wochenschr 131: 93-100

6. Dumoulin A, Hill GS, Montseny JJ, Meyrier A (2003) Clinical and morphological prognostic factors in membranous nephropathy: significance of focal segmental glomerulosclerosis. Am J Kidney Dis 41: 38-48 
7. Ginsberg YM, Chang BS, Matarese RA, Garella S (1983) Use of single voided urine samples to estimate quantitative proteinuria. N Engl J Med 309: 1543-1546

8. Greenberg A, Cheung AK, Falk RJ et al. (2005) Primer on kidney diseases: Elsevier Saunders, Mosby, pp 149-278

9. Gubler MC (2003) Podocyte differentiation and hereditary proteinuria/nephrotic syndromes. J Am Soc Nephrol 14 [Suppl 1]: S22-26

10. Hotchkiss H, Chu TT, Hancock WW et al. (2006) Differential expression of profibrotic and growth factors in chronic allograft nephropathy. Transplantation 81: 342-349

11. Marti HP, Hertig A, Mougenot B, Rondeau E (2003) Glomerulopathien. Schweiz Med Forum 46: 11081117.

12. Nortier JL, Debiec H, Tournay Y et al. (2006) Neonatal disease in neutral endopeptidase alloimmunization: lessons for immunological monitoring. Pediatr Nephrol 21: 1399-1405

13. Peterson KS, Huang JF, Zhu J et al. (2004) Characterization of heterogeneity in the molecular pathogenesis of lupus nephritis from transcriptional profiles of laser-captured glomeruli. J Clin Invest 113: 1722-1733

14. Remuzzi G, Ruggenenti P (2006) Overview of randomised trials of ACE inhibitors. Lancet 368: 555556

15. Ruggenenti P, Schieppati A, Remuzzi G (2001) Progression, remission, regression of chronic renal diseases. Lancet 357: 1601-1608

16. Thurig C, Zenhausern R, Marti HP, Landtwing B (2002) Renal involvement in malignant tumors. Ther Umsch 59: 131-137

17. Tomson CR (2000) Recent advances: nephrology. Bmj 320: 98-101

18. Tryggvason K, Patrakka J, Wartiovaara J (2006) Hereditary proteinuria syndromes and mechanisms of proteinuria. N Engl J Med 354: 1387-1401

19. USRDS (2006) Annual data report: incidence and prevalence of ESRD.http://www.usrds.org, United States Renal Data System

20. van den Berg JG, Weening JJ (2004) Role of the immune system in the pathogenesis of idiopathic nephrotic syndrome. Clin Sci (Lond) 107: 125-136

\section{Preisverleihungen 2006}

\section{Franz-Volhard-Medaille, Franz-Volhard-Preis}

Die Franz-Volhard-Medaille als höchste Auszeichnung der Gesellschaft für Nephrologie wurde im Jahr 2006 an Prof. Dr. Heini Murer aus Zürich vergeben.

Der am Universitätsklinikum Aachen tätige Prof. Dr. Peter R. Mertens wurde mit dem Franz-Volhard-Preis 2006 der Gesellschaft für Nephrologie und des Deutschen Stifterverbandes für Nierenforschung ausgezeichnet. Damit wurden seine Forschungen zu den Aktivierungswegen und extrazellulären Funktionen des Y-box Proteins-1 (YB-1) und dessen Potenzial als therapeutisches Zielprotein und prognostischer Marker für glomeruläre Erkrankungen gewürdigt.

\section{Quelle: Gesellschaft für Nephrologie e. V., www.nierengesellschaft.de}

\section{Nils-Alwall-Preis}

Für seine Untersuchungen zu der Frage, warum das Bauchfell bei der Peritonealdialyse geschädigt wird, ist Dr. Vedat Schwenger, Leitender Oberarzt in der Medizinischen Universitätsklinik Heidelberg, mit dem Nils-Alwall-Preis 2006 der Deutschen Gesellschaft für Nephrologie ausgezeichnet worden. Die besonders schonende und effektive Peritonealdialyse wird vor allem bei Kindern und Jugendlichen eingesetzt. Die Untersuchungen Schwengers haben den zugrunde liegenden Mechanismus aufgeklärt und in einem Mausmodell nachgewiesen, wie diesen Schäden vorgebeugt werden kann. Die Untersuchungsergebnisse wurden im Journal of the American Society of Nephrology (2006; 17(1):199-207) publiziert.

\section{Bernd Tersteegen-Preis}

Der Bernd Tersteegen-Preis 2006 ging nach Jena. Der Verband Deutsche Nierenzentren der DDnÄ e. V. ehrte damit Professor Dr. Gunther Wolf für eine herausragende wissenschaftliche Arbeit. Die Jury würdigte die Forschungsarbeit des Jenaer Wissenschaftlers als „zukunftsweisend und von hoher Qualität. Der Direktor der Klinik für Innere Medizin II am Universitätsklinikum Jena hatte den Ein- fluss des gefäßverengenden, körpereigenen Botenstoffs Angiotensin II auf entzündliche Nierenerkrankungen untersucht. Er konnte dabei zeigen, dass dieser blutregulierende Botenstoff eine Aktivierung des natürlichen Immunsystems bewirken kann, indem er spezielle Rezeptoren stimuliert. Diese durch das Zusammespiel von Angiotensin II und den stimulierten Rezeptoren verursachte Aktivierung des Immunsystems könnte Einfluss auf jene Autoimmunreaktionen haben, die zu Nierenerkrankungen führen.

\section{Georg Haas-Preis}

Der Verband Deutsche Nierenzentren der DDnÄ e. V. will mit der Vergabe des Georg Haas-Preises den wissenschaftlichen Nachwuchs fördern. Der Georg Haas-Preis 2006 wurde aufgrund der hohen Qualität von zwei eingereichten Arbeiten geteilt. Dr. Kerstin Benz aus Erlangen wurde für die Arbeit „In vitro Untersuchungen zur Adsorption und Filtration von rekombinantem Hirudin: Vergleich unterschiedlicher Hämodialysatoren" geehrt. Dr. Yingrui Wang-Rosenke aus Berlin erhielt die Auszeichnung für die Arbeit „Nitric oxide-cGMP signal transduction in injury, matrix expansion and progression of anti-thy1induced renal disease of the rat".

\section{Quelle: Verband Deutsche Nierenzentren derDDnÄ e. V., www.ddnae.de}

\section{Bernd Schönberger-Preis}

Erstmalig wurde der Bernd Schönberger-Preis des Arbeitskreises Nierentransplantation der Deutschen Gesellschaft für Urologie verliehen. Die Auszeichnung ging zu gleichen Teilen an die Arbeitsgruppen um Dr. Fuller (Universitätsklinikum Charité Berlin, Campus Mitte) und Dr. Meyer (Universitätsklinikum Lübeck). Der Preis geht zurück auf den 2005 verstorbenen Professor Dr. Bernd Schönberger, der sich auf dem Gebiet der Nierentransplantation engagiert hat und als Leiter der Abteilung Nierentransplantation an der Klinik für Urologie der Charité Berlin aktiv am Aufbau und an der Fortentwicklung des Nierentransplantationsprogrammes beteiligt war.

Quelle: Arbeitskreis Nierentransplantation derDGU e. 